\title{
Enabling DTN-based Data Offloading in Urban Mobile Network Environments
}

\author{
Younghwan Go, YoungGyoun Moon, and KyoungSoo Park \\ Department of Electrical Engineering, KAIST \\ \{yhwan, ygmoon\}@ndsl.kaist.edu, kyoungsoo@ee.kaist.ac.kr
}

Recent developments in smartphones and mobile devices have brought a tremendous growth in the mobile data communication. The mobile traffic volume is predicted to consume 10.8 Exabytes per month by 2016, 18x increase from that of 2011 [1]. While the next-generation mobile technologies such as Long Term Evolution (LTE) are being aggressively deployed, they are unlikely to catch up with the explosive demands. One promising solution is to opportunistically offload the bandwidth-limited mobile data transfer to the wired Internet via Wi-Fi while using the mobile networks as a backup medium to meet the transfer deadline with delay-tolerant networking (DTN) [2]. However, deploying DTN faces numerous barriers since existing applications do not seamlessly handle network delays and disruptions caused by human mobility.

In this paper, we propose a practical DTN architecture in city mobile network environments. Existing DTN designs assume that most applications are delay and disruption-tolerant, and that the network infrastructure will handle the heterogeneity of networks by utilizing the gateways and convergence layers. However, this is infeasible since it requires a significant modification to the current network framework. Instead, we apply DTN to Wi-Fi data offloading with minimal change by using DTP [3] from our previous work, which provides automatic data transfer resumption after physical network disconnections. We then introduce DProx, which supports DTP to communicate with the client to mask the mobile host's delays and disruptions to the servers.

DTP is a disruption-tolerant transport layer protocol that transparently masks network disruptions from the application layer. DTP provides an illusion of continued, but delayed connection to the applications when the underlying network is unavailable by binding the connection on an unique flow ID. However, even if the connection were to be maintained, majority of mobile applications are based on user interactions in real-time and do not support long delays. Moreover, some middle-boxes (e.g., standard Web cache) do not allow delays and close idle connections for efficient bandwidth multiplexing. We address this problem by introducing a novel disruption-tolerant proxy called DProx, which utilizes DTP to hide the mobile client's disruption and temporarily stores client's data until the complete data can be transmitted in a single connection with a server without any delay (Figure 1).

Permission to make digital or hard copies of all or part of this work for personal or classroom use is granted without fee provided that copies are not made or distributed for profit or commercial advantage and that copies bear this notice and the full citation on the first page. To copy otherwise, to republish, to post on servers or to redistribute to lists, requires prior specific permission and/or a fee.

CFI'12, September 11-12, 2012, Seoul, Korea

Copyright 2012 ACM 978-1-4503-1690-3/12/09 ...\$15.00.

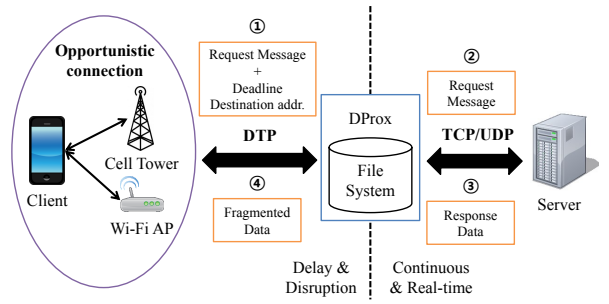

Figure 1: DTN architecture in urban mobile network environments.

There are several challenges in developing DProx. Introducing a proxy that acts on behalf of the client raises a critical security and privacy issue in user authentication. This can be solved by authenticating the trusted DProx before the client initiates a connection via a trusted certifying authority (CA). DProx also requires a DTN-aware scalable file system design for storing and fetching data from multiple concurrent connections while utilizing the deadline. We thus design a LFS-like file system, in which client's data with larger deadline are stored in disk until clients with small deadline are first handled. We also design a deadline monitoring system, which deletes implicit data when it detects an expiration.

DProx enables a number of new applications such as a publishand-subscribe system where the subscribed users allow delays to receive published feeds, and a network resource multiplexer which postpones transferring the mobile data untill the network load becomes low to spread the traffic usage over the time axis in the backend Internet infrastructure. This "deadline-based network transfer" will greatly help in reducing the non-interactive traffic at peak times, and can increase the effective bandwidth for the mobile users.

We propose a new DTN architecture that provides an illusion of continuous and real-time data transfer to the destination hosts by transparently masking the client's DTN characteristics. Currently, we have implemented a HTTP-enabled DProx and have begun collecting real-life traces from users to analyze the human mobility patterns in DTN environments. As a future work, we plan to design an algorithm for determining an appropriate deadline for the users, and develop a practical DTN-based data offloading system.

\section{Acknowledgements}

This research was supported by KCA (Korea Communications Agency) in South Korea, KCA-2012-11913-05004.

\section{REFERENCES}

[1] CISCO. Cisco Visual Networking Index: Global Mobile Data Traffic Forecast Update, 2011-2016. Technical report, 2012.

[2] K. Fall. A Delay-Tolerant Network Architecture for Challenged Internets. In Proceedings of ACM SIGCOMM, 2003.

[3] Y. Go, Y. Moon, G. Nam, and K. Park. A Disruption-tolerant Transmission Protocol for Practical Mobile Data Offloading. In Proceedings of ACM MobiOpp, 2012 